\title{
ProtoMatch: A tool for analyzing high-density, sequential eye gaze and cursor protocols
}

\author{
CHRISTOPHER W. MYERS and MICHAEL J. SCHOELLES \\ Rensselaer Polytechnic Institute, Rensselaer, New York
}

\begin{abstract}
ProtoMatch is a software tool for integrating and analyzing fixed-location and movement eye gaze and cursor data. It provides a comprehensive collection of protocol analysis tools that support sequential data analyses for eye fixations and scanpaths as well as for cursor "fixations" (dwells at one location) and "cursorpaths" (movements between locations). ProtoMatch is modularized software that integrates both eye gaze and cursor protocols into a unified stream of data and provides an assortment of filters and analyses. ProtoMatch subsumes basic analyses (i.e., fixation duration, number of fixations, etc.) and introduces a method of objectively computing the similarity between scanpaths or cursorpaths using sequence alignment. The combination of filters, basic analyses, and sequence alignment in ProtoMatch provides researchers with a versatile system for performing both confirmatory and exploratory sequential data analyses (Sanderson \& Fisher, 1994).
\end{abstract}

The increased use of eye tracking technologies in conjunction with real-world task environments and scaled worlds (Gray, 2002) has accelerated the need to accurately incorporate and evaluate eye movement protocols with cursor protocols. ProtoMatch is a software tool for analyzing and exploring such sequential data. It incorporates eye gaze positions and saccades, cursor locations and movements, and mouse clicks into a unified stream of parallel sequential data derived from log files produced by users behaving in task environments.

ProtoMatch was developed to analyze high-density sequential protocol data, defined here as eye gaze and cursor positions continuously sampled at high rates (e.g., 60 samples/sec or more) throughout a given task. Such highdensity data are also high-definition - providing nearly complete records of human-environment interactions. High-density, high-definition data afford insights into complex human behavior. Collecting high-density protocol data facilitates analyses of behavior occurring at the level of primitive behavioral actions (e.g., eye and cursor movements, fixations, dwells, and cursor clicks; see, e.g., Anderson, 2002; Card, Moran, \& Newell, 1983; Newell $\&$ Card, 1985). Such primitive actions provide atomic criteria for cognitive modelers of all types (GOMS, ACT-R, Soar, Epic, etc.) to use when comparing their models and human data.

This work was supported by grants from the Air Force Office of Scientific Research (AFOSR F49620-03-1-0143) and the Office of Naval Research (ONR N000140310046) to Wayne D. Gray. The authors thank Wayne D. Gray, Markus Guhe, and Hansjörg Neth for their valuable comments on earlier drafts of this report. A special thanks to Chris R. Sims and Wayne D. Gray for software name suggestions. Correspondence concerning this article should be addressed to C. W. Myers, Cognitive Science Department, Rensselaer Polytechnic Institute, CogWorks Laboratories, 110 8th Street, Troy, NY 12180 (e-mail: myersc@rpi.edu).
Although verbal protocols and social interactions are valuable in understanding goal-directed behavior (Ericsson \& Simon, 1980), ProtoMatch was not developed with such data in mind. Although it may be possible to apply ProtoMatch to such data, such applications are not the focus of the present report. Rather, ProtoMatch was developed to facilitate the analysis of high-density fixedlocation and movement protocols from two sourceseyes and cursors (see Table 1). Fixed-location protocols are defined here as relatively discrete, easily separable protocols (such as mouse clicks), whereas movement protocols are relatively continuous (such as eye movements). Both fixed-location and movement protocols will be discussed in more detail in the upcoming sections relative to their source type (eye or cursor).

Pioneering work on the analysis of fixed-location cursor protocols (see Fu, 2001; Ritter \& Larkin, 1994) and of fixed-location eye gaze protocols (Salvucci \& Anderson, 2001) has demonstrated the benefit of analysis automation. The present work continues in the tradition of automating aspects of protocol analyses, but it differs from previous work in two important ways: (1) ProtoMatch incorporates four types of protocols (movement and fixed-location cursor and eye gaze protocols) with beneficial automation and (2) it is reusable software that will facilitate analyses not envisioned by its creators.

\section{PROTOCOLS}

\section{Cursor Protocols}

Cursor protocols can be divided into two types: fixedlocation and movement (see Table 1). We define movement cursor protocols as the path a cursor moves over a period of time, or a cursorpath. Such information can, for example, be used to determine cursor gestures (Moyle \& Cockburn, 2003). 
Table 1

Data Source $\times$ Data Type Matrix

\begin{tabular}{lll}
\hline \multirow{2}{*}{ Data } & \multicolumn{2}{c}{ Data Type } \\
\cline { 2 - 3 } Source & \multicolumn{1}{c}{ Movement } & \multicolumn{1}{c}{ Fixed Location } \\
\hline Eye & Scanpaths and saccades & Fixations or dwells \\
Cursor & Cursorpaths and movements & Fixations, dwells, or clicks \\
\hline
\end{tabular}

Fixed-location cursor protocols can be divided into three subtypes: cursor clicks within an interface, the steady positioning of a cursor (fixations), and multiple consecutive cursor fixations at approximately the same location (dwells). We have found no mention of cursor fixations or dwells in the literature; however, cursor clicks have been regularly used to analyze behavior in task environments (Card et al., 1983; Fu, 2001; Payne, Bettman, \& Johnson, 1993; Simon, 1999; Todd \& Benbasat, 1994, 1999, 2000).

There are currently two major approaches to analyzing sequential fixed-location cursor protocol data (i.e., cursor clicks): (1) trace-based and (2) top-down. Tracebased approaches (see Ritter \& Larkin, 1994, for an example) relate human protocol traces to predictions of sequential behavior produced by a theoretical model of behavior. Top-down approaches match human protocols to a hierarchical goal decomposition of the environment (see Fu, 2001, for an example).

A limitation of the top-down approach is the "generate and test" cycle typically necessary for finding the most restrictive goal-subgoal task hierarchy that fits the subjects' data best. As a result, analyses are restricted to focusing on either novice or expert behavior and may not capture patterns indicative of users switching from a novice to an expert strategy (Siegler \& Stern, 1998). A limitation of the trace-based protocol analysis advocated by Ritter and Larkin (1994) is that one must first develop an accurate computational cognitive model. If the model's trace does not adequately fit subjects' behavioral traces, the researcher must generate a new model and test it against subjects' data.

High-density cursor data have been used to gain insight into sequential behavior underlying goal achievement in task environments (see $\mathrm{Fu}, 2001$, for an excellent example). To gain useful insights with minimal costs to the researcher, aspects of combining protocol samples and protocol analyses must be automated or semiautomated (Fu, 2001; Ritter \& Larkin, 1994; Salvucci \& Anderson, 2001). A central focus of ProtoMatch development has been to adopt the usefulness of automation without the necessity of generate-and-test cycles associated with previous work (Fu, 2001; Ritter \& Larkin, 1994).

\section{Eye Gaze Protocols}

Fixed-location cursor protocols do not tell the entire behavioral story; that is, eye gaze protocols can also significantly contribute to understanding goal-directed behavior in task environments. For instance, an interactive problem-solving task (e.g., the Tower of Hanoi) can be solved visually prior to any fixed-location cursor protocols (i.e., cursor clicks). In such cases, fixed-location cursor protocols do not capture the process of finding the solution to the problem; instead, they capture the solution to the solved problem. Capturing and incorporating movement and fixed-location eye gaze protocols (see Table 1) into analyses will allow researchers to determine not only when, but also how, problems are solved and decisions made using eye movements, how plans are orchestrated via eye movements, and how movement and fixed-location cursor and eye gaze protocols are interleaved and executed in parallel.

Typical data acquired from eye trackers are pupil diameter and foveal position in the form of two-dimensional Cartesian coordinates. Log files containing samples from eye trackers can be processed to yield fixed-location and movement eye gaze protocols. Fixed-location protocols can be characterized as fixations (stable positioning) or dwells (multiple fixations at approximately the same location). Movement protocols can be characterized as saccades (high-velocity movements between fixations/ dwells) or scanpaths, which are constructed by temporally linking fixations and/or dwells (see Table 1).

To counter the unwieldy amounts of data produced from eye tracking, interest in developing automated analyses focusing on fixations, dwells, saccades, and scanpaths has been increasing (Goldberg \& Kotval, 1999; Ponsoda, Scott, \& Findlay, 1995; Salvucci \& Anderson, 2001). Recent work by Salvucci and Anderson (2001) provides an example of this endeavor. The authors described and evaluated approaches to automating eye movement protocol analyses that mirror the approach of Ritter and Larkin (1994). That is, such approaches relate eye movements to the sequential predictions of a process model.

Eye movement protocols have been used to understand how images are interpreted (Yarbus, 1967), to determine how text is read (Rayner, Liversedge, White, \& Vergilino-Perez, 2003), to verify information that is critical in making decisions (Lohse \& Johnson, 1996), and to infer strategies involved in visual search (Shen, Reingold, \& Pomplun, 2000). However, drawing such conclusions about goal-oriented behavior using eye gaze protocols typically has involved significant added effort. Scanpath analyses have suffered from a lack of objectivity. For instance, Yarbus (1967) demonstrated scanpath differences by asking the same subject different questions about the same image. Differences were determined through subjective analysis: If two scanpaths appeared to be different, they were deemed to be different.

Some progress has been made with objectively analyzing scanpaths (Goldberg \& Kotval, 1999; Lipps \& Pelz, 2004; Ponsoda et al., 1995). Goldberg and Kotval (1999) have provided two main categories of analyses: measures of search and measures of processing. Measures of search include calculating such relevant factors 


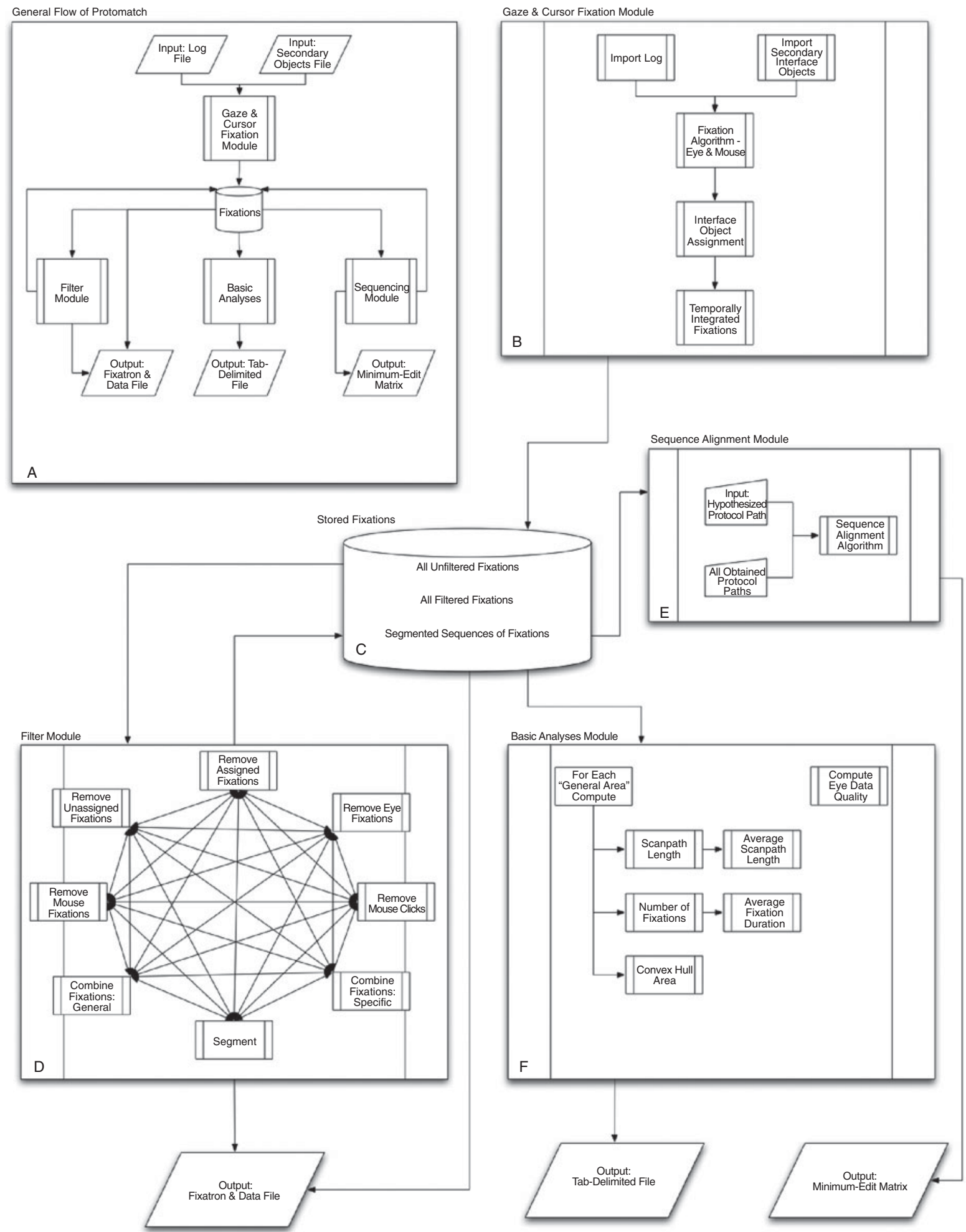

Figure 1. The process flow of ProtoMatch. Box A is a general view of the flow of ProtoMatch. Box B is the process flow for the gaze and cursor fixation module, and Box $C$ is the fixation repository. Boxes D-F are, respectively, the filter, sequence alignment, and basic analyses modules. 
as scanpath lengths and durations, convex hull areas for each scanpath, transition matrices, and the number of saccades and their amplitudes. Measures of processing include calculating the number of fixations and fixation durations and calculating the fixation/saccade ratio. Much progress has been made in eye gaze protocol analysis, but there has yet to be a method or system that objectively computes the similarity of two complete scanpaths or two subsections of either different scanpaths or the same scanpath.

Unfortunately, one reason for the lack of such a system is that high-density protocol data become unwieldy and difficult to analyze as the complexity of the task and of the task environment increases. Examples of complex task environments include driving an automobile while talking on a cellular phone (Salvucci \& Macuga, 2002) or determining which aircraft on a radar screen to eliminate (Gray, Schoelles, \& Myers, 2004). Such large-scale task environments produce visually complex stimuli and complex sequential patterns of behavior that dramatically increase the difficulty of analyzing and interpreting cursor and eye gaze protocols.

\section{Protocol Paths}

ProtoMatch was developed, in part, to overcome the traditional separation of fixed-location eye gaze and cursor protocols by integrating them. Integration occurs by sequentially interleaving scanpaths and cursorpaths into a new protocol category called protocol paths. Such integration allows the researcher to analyze the complex interactions between action and perception in task environments.

Protocol paths created in ProtoMatch are single "streams" of behavioral data that represent two parallel and sequential streams of behavior: cursor data and eye gaze data. The data are parallel in that single or multiple eye gaze and cursor events may occur within the same time interval.

Protocol paths provide a high-density, high-definition record of behavior. Analyzing protocol paths promises to facilitate a more robust understanding of behavior in general, and the interaction between movement and fixedlocation eye gazes and cursor protocols in particular.

In order to make good use of protocol paths (or of scanpaths or cursorpaths alone), automated analyses need further development. One example is the ability to easily evaluate the similarity between scanpaths, cursorpaths, and protocol paths. Developing software tools for determining the similarity between protocol paths can benefit from tools developed by bioinformaticians. A family of tools used in bioinformatics focuses on finding subsequences within a larger sequence of information. The same sequencing algorithms and techniques used in bioinformatics can be applied to determine the similarity between protocol paths (as well as between cursorpaths and scanpaths).

To sum up, fixed-location cursor and eye gaze protocols have traditionally been analyzed separately. ProtoMatch overcomes this separation by combining them in protocol paths. The software contains a sequence alignment algorithm that facilitates objective behavioral-path (scanpath, cursorpath, and protocol path) comparisons and reduces the effort associated with such analyses. ProtoMatch also houses a score of automated basic analyses to reduce the time required to prepare and analyze high-density, high-definition behavioral data.

\section{OVERVIEW OF PROTOMATCH MODULES}

ProtoMatch is written in ANSI Common Lisp using the LispWorks development environment (LispWorks, Ltd.; www.lispworks.com). The software currently runs on Apple Macintosh and is in the process of being ported to other systems. ProtoMatch is composed of four core modules: the gaze and cursor fixation module, the filter module, the basic analyses module, and the sequence alignment module (see Figures $1 \mathrm{~A}-1 \mathrm{~F}$ ). ProtoMatch is modularized to promote component exchange. Each module will be discussed in-depth and made concrete through examples in the following subsections.

We will walk through the ProtoMatch modules using examples of data taken from human subjects using the Decision Making Argus Prime (DMAP) task environment (Gray et al., 2004). DMAP requires both visual search and decision-making behaviors (see Figure 2 for an example of the task interface). Although the task is somewhat complex, its description will be limited in order to keep a focus on ProtoMatch.

Very simply, the subjects' goal in DMAP in our examples is to eliminate one of a select group of targets (right panel, Figure 2) found on a simulated radar (left panel, Figure 2). Subjects have $60 \mathrm{sec}$ to choose the target with the highest value. Target values are determined by finding and clicking on an alternative in the radar. If the clicked target has the greatest value, it can be selected for elimination by clicking the corresponding "Track \#" in the table of choice alternatives (ToA; right panel). After determining and selecting the desired target for elimination, the subject clicks the Choose button (below ToA). Each subject performs many choice tasks over the course of eight 12-min scenarios. A complete record of each scenario is written to a log file for later processing by ProtoMatch and other analysis software.

\section{Gaze and Cursor Fixation Module}

Overview. ProtoMatch reads a log file of raw eye gaze and cursor samples (i.e., $x$ - and $y$-coordinates) that it parses into fixations and saccades. ProtoMatch's gaze and cursor fixation module treats eye gaze position and cursor position data nearly identically. Thus, ProtoMatch divides continuously sampled streams of data into fixedlocation events through fixation formation. Movement data, such as saccades and cursor movements, are defined as the movement and trajectory from one fixation to the next.

Mouse clicks are fixations with special status and are stored separately from their nonclick cursor fixation counterparts. System events are also stored, enabling the 


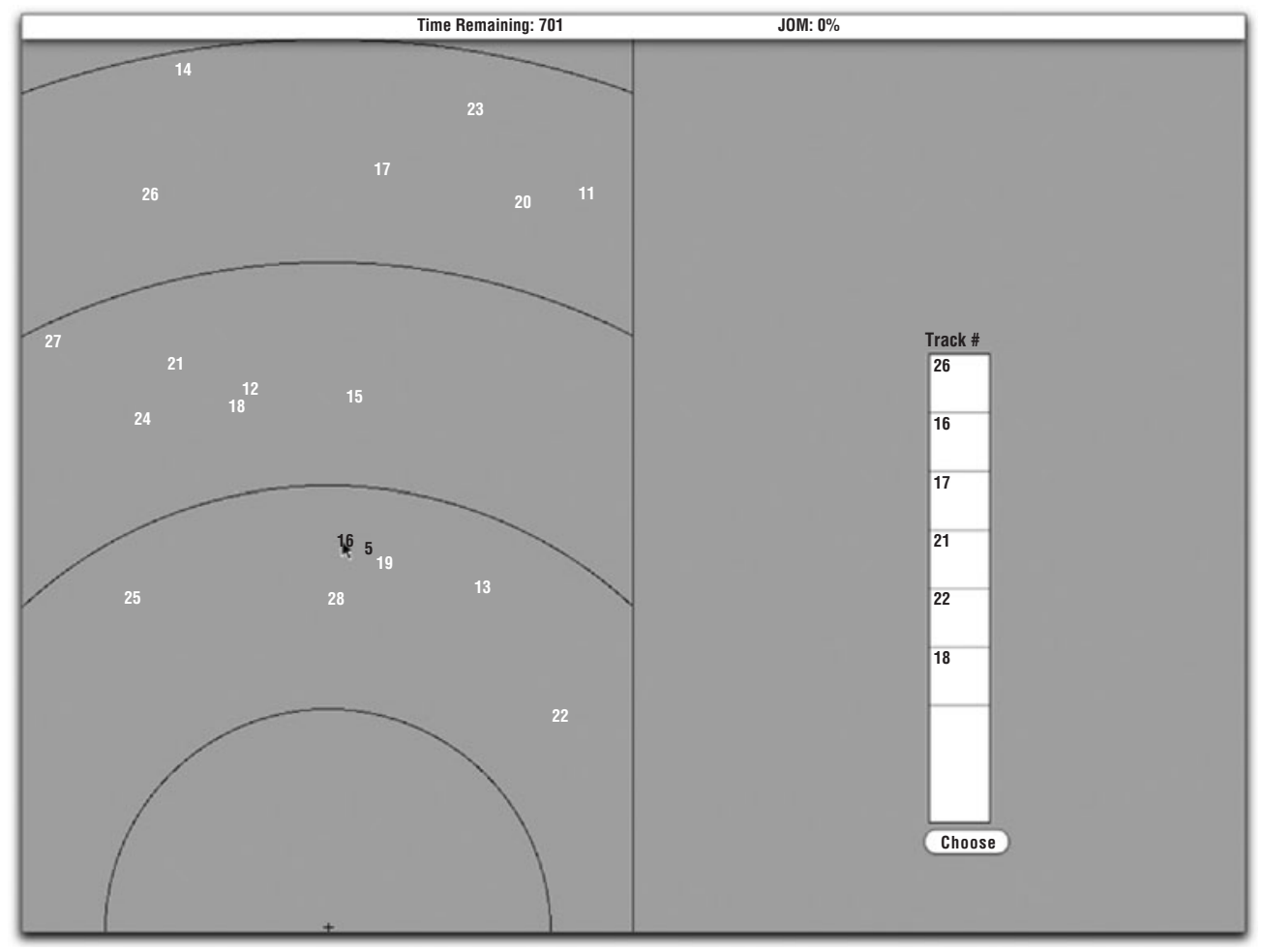

Figure 2. Decision Making Argus Prime. The left half of the display is a simulated radar screen, and the right half is the table of choice alternatives of a choice task. Target 16 has been clicked in the radar, and its current value (5) is displayed.

user to anchor analyses on logged changes in the task environment.

Eye gaze and cursor location information are read from a selected log file, beginning with the earliest recorded eye gaze or cursor location and ending with the latest. ProtoMatch's default dispersion-based fixation algorithm defines fixations as a minimum of 6 eye gaze or cursor samples within a default $2^{\circ}$-of-visual-angle window of resolution (Karsh \& Breitenbach, 1983). The window of resolution is parameterized to change the number of pixels that constitute $2^{\circ}$ of visual angle at different monitor resolutions and at a fixed viewing distance of approximately $22 \mathrm{in}$. The number of samples per fixation is parameterized to specify the minimum number of samples within the window of resolution necessary to compose a fixation.

The center of gravity of a potential fixation is an average of the Cartesian coordinates of all included eye gaze samples. Thus, the window-of-resolution center changes as the number of included gaze or cursor point samples increases. Once the window-of-resolution diameter is set (e.g., $2^{\circ}$ of visual angle), it stays constant throughout center-of-gravity repositioning (see Figure 3). If two consecutive gaze or cursor point samples fall outside the window of resolution and occur within $2^{\circ}$ of each other, the current potential fixation is aborted and a new potential fixation begins that includes the same two gaze or cursor point samples that resulted in the abortion of the previous potential fixation (see Figure 4, Box A to Box B). However, if two gaze or cursor points fall outside of the window of resolution and are not within $2^{\circ}$ of visual angle, the latest sample begins a new potential fixation (see Figure 4, Box A to Box C to Box D). Following Karsh and Breitenbach (1983), fixation durations are set, by default, to a minimum of $100 \mathrm{msec}$ ( 6 samples at a sampling rate of $60 \mathrm{~Hz}$ and 24 samples at a sampling rate of $240 \mathrm{~Hz}$ ). For each fixation, ProtoMatch stores its duration, center of gravity (in Cartesian coordinates), beginning and end times, the number of eye gaze or cursor samples, and the interface object fixated, described below.

Once a fixation has been determined, ProtoMatch assigns an interface object to the fixation. This process is similar to the target tracing method discussed by Salvucci and Anderson (2001); however, ProtoMatch assigns interface objects to both eye gaze and cursor fixations/dwells. Interface objects are any interface items of interest that can be moused over, clicked, or gazed at and whose information is included in the log file or a "secondaryobject" file (see the Appendix). Such interface objects are assigned by determining the object with the smallest difference between the fixation's center-of-gravity location and an object's location, for all objects within $2^{\circ}$ of visual angle of the fixation's center of gravity. In the case of a tie in the minimum distance between two or more interface objects, or for fixations occurring in an area where no interface object is within $2^{\circ}$ of visual angle, the 
fixation is assigned an empty interface object with an appropriate label (i.e., "unknown"). After all data have been read in, run through the fixation algorithm, and assigned an appropriate interface object, all fixations are temporally sorted from the earliest to the latest, thus providing a high-definition record of user behavior.

Example of use. ProtoMatch is used via a graphical user interface (GUI; see Figure 5 for a current version). Because the ProtoMatch GUI is constantly being modified, Figure 5 depicts ProtoMatch's current functionality and not a final version of the interface.

To analyze DMAP data, an example user first loads a single DMAP log file and a single secondary-object file that includes interface objects left out of the log file (see the Appendix for descriptions of log and secondaryobject files and their necessary formats). Next, the user executes the fixation algorithm, interface object assignment, and update functions via the ProtoMatch interface (see "Initialization Utilities," Figure 5). The eye gaze and cursor fixation algorithm combines data read in from the $\log$ and secondary-object files to determine fixations, as described above. Once a fixation is determined, ProtoMatch attempts to assign the closest interface object within $2^{\circ}$ of visual angle.

Once fixations are established, ProtoMatch sorts the list of all fixations and their associated interface objects into temporal order. At this point, the user may check the quality of the eye protocol data or produce a static display of all fixations (eye gaze and cursor) and mouse clicks (see Figure 6).

\section{Filter Module}

Overview. The filter module simultaneously provides a means of systematic data reduction and enables similarity comparisons between protocol paths (hypothesized or obtained empirically) without the need of a complex formal grammar. Currently, eight filters have been implemented as a means to manipulate the protocol data. Table 2 presents the filters and a brief description of their associated functions.

The Segment filter allows the user to "split" the temporal sequence of fixations into task-specific segments. For instance, if a single log file contains multiple trials of an experimental task (as occurs in most within-subjects experimental designs), the segmentation filter allows the user to divide the protocols into instances of withinsubjects trials.

Reducing data using the filters described in Table 2 increases the processing speed of the sequencing module by combining fixations into dwells or removing fixations of little or no interest to the researcher. Using the filters also provides a means for the ProtoMatch user to differentiate general and specific levels of analysis. For instance, if the user is interested in specific interface objects within a general area of the task environment, the Combine Fixations: Specific filter combines all consecutive fixations to a specific object into a single dwell. However, if the researcher is only interested in the movement of the cursor and gaze between general areas of the task environment, the Combine Fixations: General filter combines all consecutive fixations within a general area

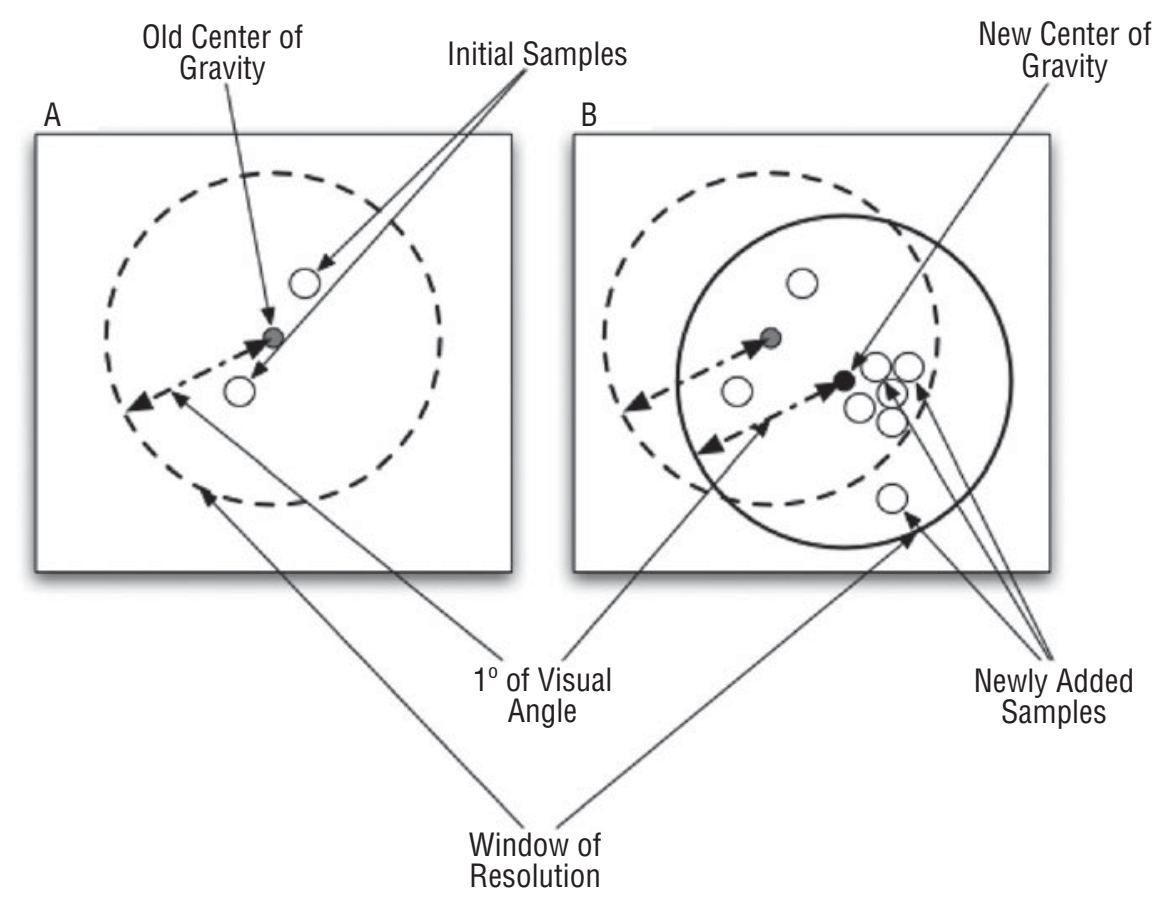

Figure 3. Movements of fixation center of gravity with the inclusion of new gaze or mouse point samples. (A) Samples at time $N$. (B) Samples at time $N+1$. Note that fixation radius does not change as fixation center of gravity shifts. 


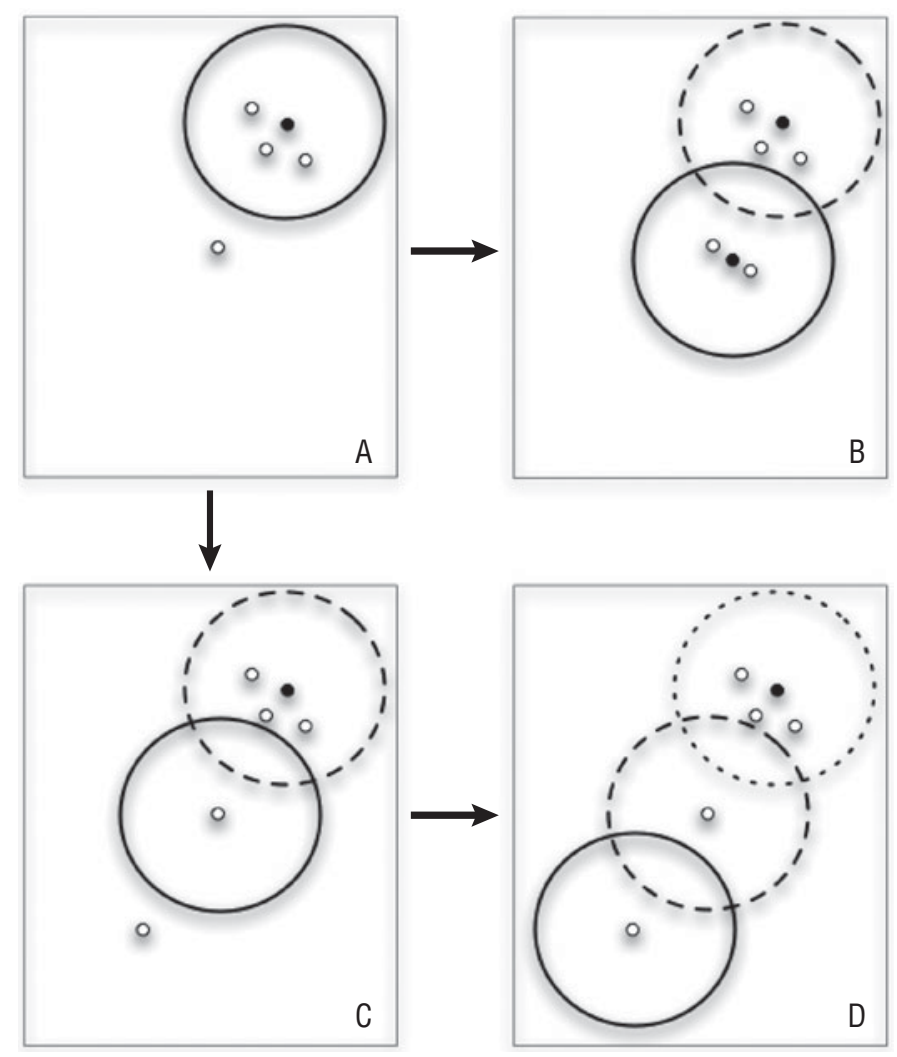

Figure 4. A detailed example of defining a fixation. (A) occurs at time $N$ and contains a potential fixation with three samples (white circles) inside the window of resolution and one outside. Throughout the figure, a black dot represents a potential fixation's center of gravity. When only one sample is present, it is the new potential fixation's center of gravity. Aborted potential fixations are denoted by dashed circles and are irrelevant to potential fixations. (B) and (C) are two possibilities for time $N+$ 1: (B) two samples within $2^{\circ}$ of visual angle of each other, creating a new potential fixation; (C) samples not within $2^{\circ}$ of visual angle of each other. (D) occurs at time $N+2$ and extends this example by demonstrating a new potential fixation created for the latest sample.

into a single superdwell. Filters may be used in any order and can only be used once per analysis (see Figure 1D). ProtoMatch also contains a Revert function, allowing the researcher to return the data to the state prior to any filtering (see Figure 5).

Example of use. Once the example ProtoMatch user has acquired all fixations, the protocol data are ready for filtering. The protocols are now eye fixations, mouse fixations, and mouse clicks and can be filtered in several ways. For instance, the user analyzing a DMAP scenario could wish to remove all fixations that are not assigned an interface object, and thus would use the ":Remove no-object fixations" capability. The ProtoMatch user could also wish to remove mouse fixations and would accomplish this by using ":Remove Mouse fixations." To re-enable the selected filters, allowing for further and different analyses on the same sequential protocols, the user could simply use the Revert function. That is, Revert brings the user back to the state just before filtering.
After removing the fixations deemed uninteresting or unnecessary, the example user collapses fixations into dwells. That is, the user combines two or more consecutive fixations on an identical interface object (e.g., Target 16 on the radar in Figure 2) by using the Combine Fixations: Specific function. Each new dwell is a combination of the center-of-gravity position, beginning time, ending time, and duration of all fixations collapsed into the new dwell. (Cursor clicks are not combined at any level.) The fixations that are used in dwell construction are automatically removed.

The ProtoMatch user then uses the Combine Fixations: General function and combines two or more consecutive dwells within the same general interface area (e.g., radar or ToA in DMAP) into a single superdwell. For example, suppose there were six consecutive eye fixations (A, B, C, D, E, and F) on the radar in Figure 2. Using the Combine Fixations: General function would collapse fixation A into B, B into C, C into D, and so forth, effectively combining fixations $\mathrm{A}$ through $\mathrm{F}$ into a 


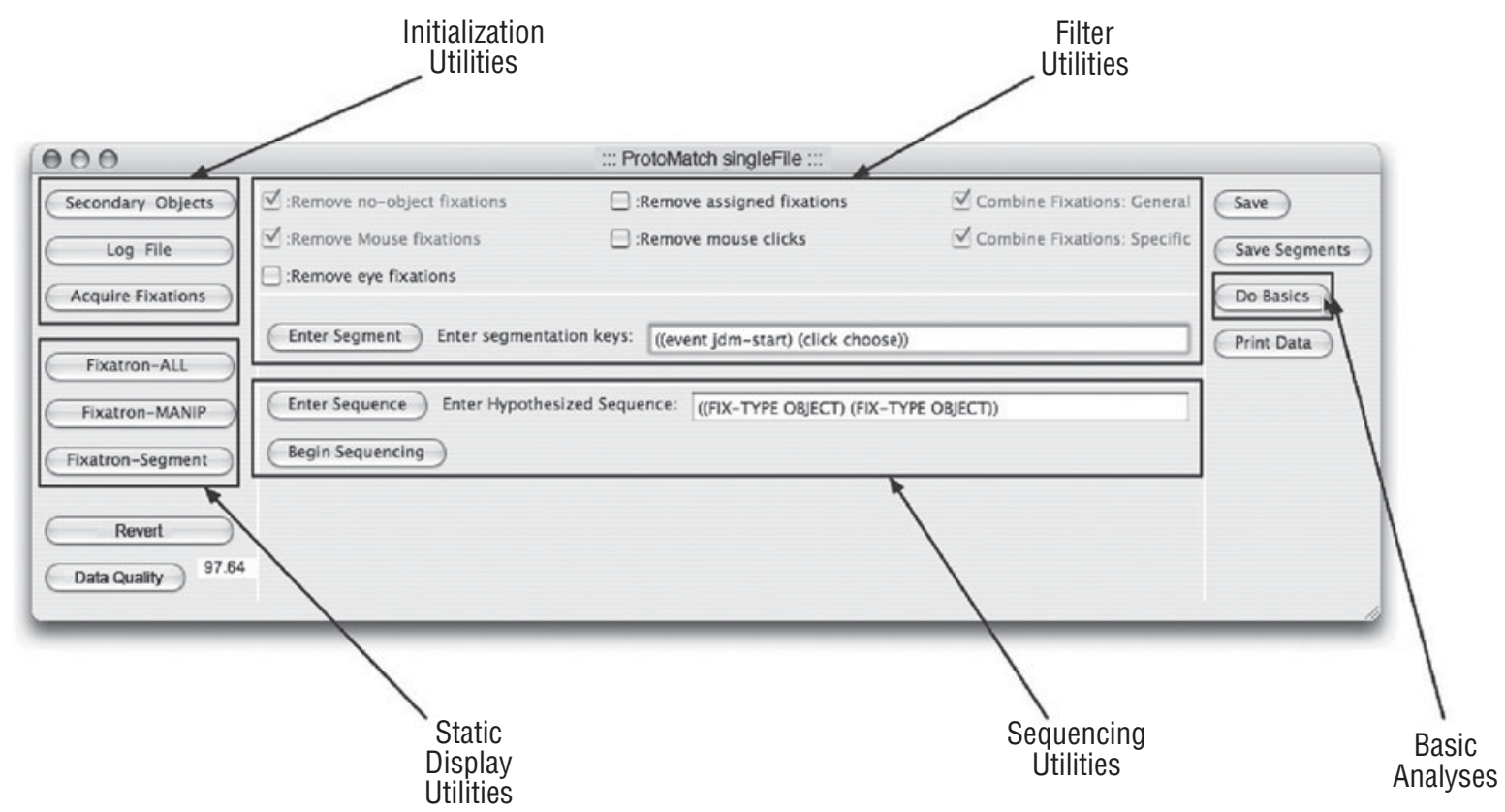

Figure 5. The ProtoMatch interface, divided into initialization utilities, filter utilities, static display utilities, sequencing utilities, and basic analyses.

single superdwell oriented on a general area of interest. Such superdwells are combined regardless of whether they were directed at the same specific targets or not. This level of combination reduces the complexity within each protocol path and provides a very general analysis of behavior.

The Combine functions use the beginning and ending times, the specific object label, and the general areas of the interface of dwells/fixations, as defined via the log file (see Appendix, Table A1, last column) or secondaryobject files (see Appendix, Table A2, column 5), to appropriately combine fixations into dwells and dwells into superdwells.

ProtoMatch users can also divide the fixations into meaningful segments that might represent repeated trials of within-subjects experimental designs. For example, DMAP subjects are given choice tasks every $60 \mathrm{sec}$ throughout a 12-min scenario; therefore, the ProtoMatch user can divide the protocols into segments of choice tasks. The user must enter segmentation keys by specifying a fixation-type/fixated-object pair representing the beginning and ending of the desired segment. System events and their associated log file tags may also be used as segmentation keys. The segmentation utilities can be used either before or after filtering.

To continue the example above, the ProtoMatch user currently analyzing DMAP data is interested in dividing the data into segments beginning with the first choice task and ending with the DMAP subject clicking on the Choose button. Therefore, the user enters the segmentation keys "((event jdm-start) (click choose))" into the segmentation text input field and presses the Enter Seg- ment button. The "event" key represents a system event and is general to ProtoMatch. However, the "jdm-start" key is the log file tag for the beginning of a new choice task and is specific to DMAP. The user can now view static displays of all segments by repeatedly using the Fixatron-Segment function (see "Static Display Utilities," Figure 5).

Practically speaking, the ability to view static displays of the segmented fixations may provide enough detailed information to derive hypotheses. That is, the ability to view segmented fixations would provide an initial step in exploratory sequential data analyses (Sanderson \& Fisher, 1994). The example ProtoMatch user might then save these segments to tab-delimited files using the Save Segments function for further analyses with other data analysis software. Such files contain the fixation information (i.e., fixation type, begin time, end time, $x$ - and $y$-coordinates of the center of gravity, fixation duration, and assigned interface object information).

\section{Basic Analysis Module}

Overview. The basic analysis module provides the ProtoMatch user with a high-level perspective of the fixed-location and movement eye gaze and cursor protocol data. The basic analyses are a select group of measures discussed in Goldberg and Kotval (1999). Analyses on movement protocols include the length of a scanpath per segment (gaze or cursor, in pixels), the average length of scanpaths per segment (gaze and cursor, in pixels), and the convex hull area per segment (gaze and cursor, in pixels). Analyses on fixed-location protocols include the number of fixations per segment (gaze and cursor) and 


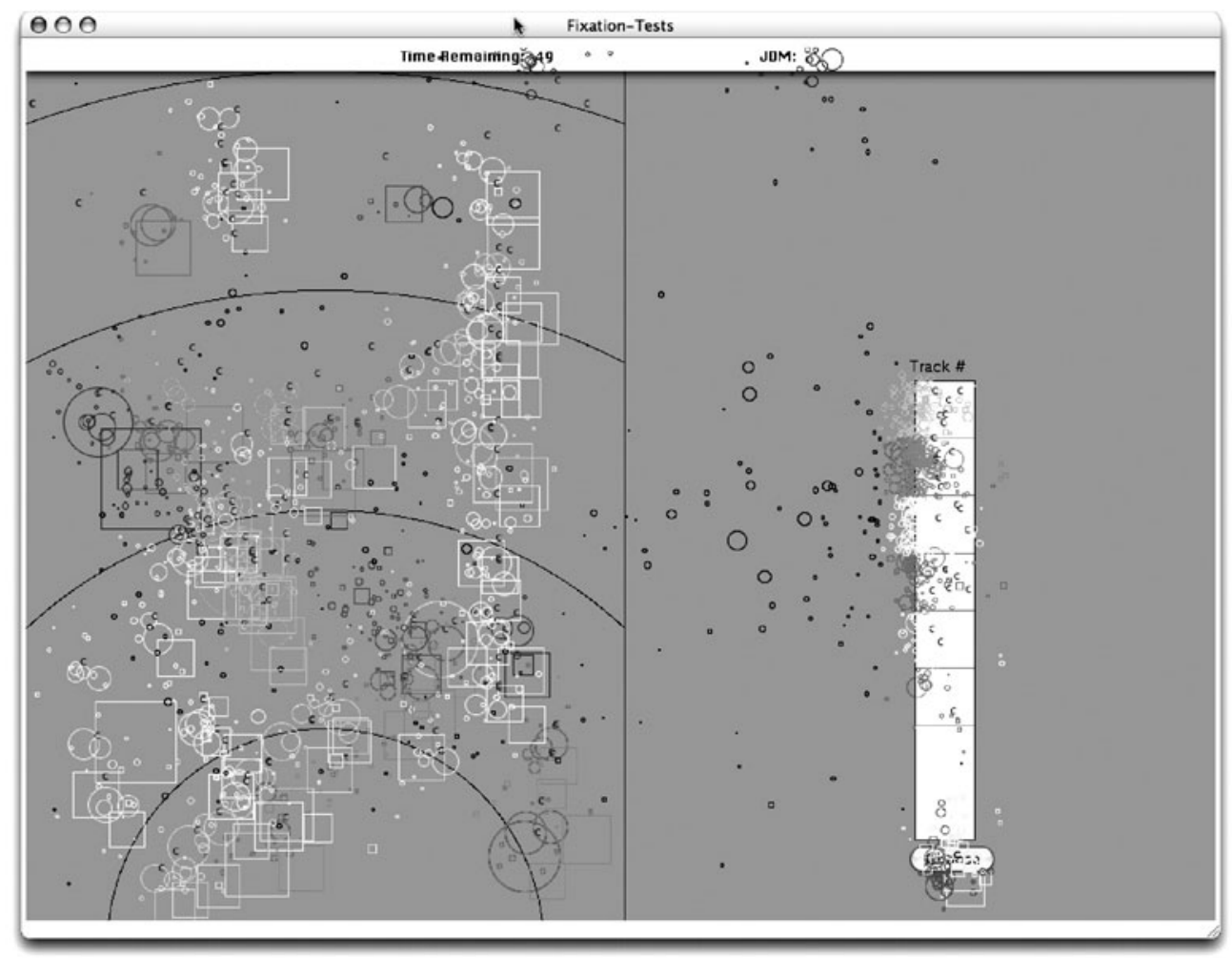

Figure 6. An example of static visual output derived from clicking the Fixatron-ALL button. Light and dark gray shapes represent fixations with assigned interface objects, and black shapes represent fixations without assigned interface objects. Circles represent eye fixations, squares cursor fixations, and $c s$ mouse clicks. Areas within circles and squares represent relative fixation durations. The data are from one scenario for Participant S2.

the average fixation duration per segment (gaze and cursor). The basic analyses can be used on either filtered or unfiltered data.

The basic analyses are computed for all general interface areas as defined by the ProtoMatch user via log files or secondary-object files (see the Appendix). Also, if segmentation is not used, the basic analysis function treats an entire log file as a single segment. For a comprehensive discussion of ProtoMatch's basic analyses, see Goldberg and Kotval (1999). To run the basic analyses, the user simply executes the Do Basics function. All analyzed data from the basic analyses are saved in a newly created file named "basicsNEW.txt" within the same directory from which the log file was originally chosen.

Example of use. After executing gaze and cursor fixation functions, filtering the data, and segmenting the protocols exemplified above for two DMAP subjects, the example ProtoMatch user runs basic analyses. A portion of the data is presented in Table 3 after the user has computed means and standard deviations from the ProtoMatch output.

\section{Sequence Alignment Module}

Overview. The sequence alignment module allows ProtoMatch users to objectively compute the similarity between two segments (see Figure 1E). The core of this module is a sequence alignment algorithm. The algorithm takes a hypothesized (or obtained) protocol path as input ( $\mathrm{PP}_{\mathrm{H}}$ for convenience). $\mathrm{PP}_{\mathrm{H}}$ is then aligned to an obtained protocol path, filtered or unfiltered $\left(\mathrm{PP}_{\mathrm{O}}\right)$. The algorithm returns the minimum number of edits necessary to change $\mathrm{PP}_{\mathrm{H}}$ into $\mathrm{PP}_{\mathrm{O}}$.

The alignment algorithm works like a spellchecker, determining the best fit based on the minimum-edit distance between two segments, hypothesized or obtained. The minimum-edit distance is determined by finding the minimum number of insertions, deletions, and replacements (edits) necessary to change $\mathrm{PP}_{\mathrm{H}}$ into $\mathrm{PP}_{\mathrm{O}}$. For example, to change FIREMAN $\left(\mathrm{PP}_{\mathrm{H}}\right)$ into POLICEMAN $\left(\mathrm{PP}_{\mathrm{O}}\right)$, the algorithm's solution would (1) insert a $P$ to the left of the $F$; (2) insert $O$ to the left of the $F$; (3) replace $F$ with $L$; and (4) replace $R$ with $C$. Therefore, the minimumedit distance for changing FIREMAN into POLICEMAN is 4 .

Replacing the letters in the FIREMAN-POLICEMAN example with individual protocols and their associated objects (i.e., $F=$ [Eye Data Object 1], $I=$ [Eye Data Object 2], $R=$ [Mouse Data Object 1], $E=$ [Mouse Click Object 1], etc.) from a protocol path provides an example of how behavioral data are used by the sequencing algorithm. The sequence alignment algorithm is similar to the minimum-edit algorithms used by Salvucci and Anderson (2001) and $\mathrm{Fu}$ (2001) and discussed by Card et al. 
Table 2

ProtoMatch Filters and Their Associated Functions

\begin{tabular}{|c|c|c|}
\hline & Filter & Function \\
\hline 1 & Remove Unassigned Fixations & $\begin{array}{l}\text { Eliminates fixations that have not been assigned } \\
\text { an interface object }\end{array}$ \\
\hline 2 & Remove Assigned Fixations & $\begin{array}{l}\text { Eliminates fixations that have been assigned an } \\
\text { interface object }\end{array}$ \\
\hline 3 & Remove Eye Fixations & Eliminates all eye fixations \\
\hline 4 & Remove Mouse Fixations & Eliminates all mouse fixations \\
\hline 5 & Remove Mouse Clicks & Eliminates all mouse clicks \\
\hline 6 & Segment & $\begin{array}{l}\text { Divides data into meaningful segments via } \\
\text { user-entered beginning and ending information }\end{array}$ \\
\hline 7 & Combine Fixations: General & $\begin{array}{l}\text { Combines two or more consecutive fixations } \\
\text { occurring on the same general interface area of } \\
\text { interest }\end{array}$ \\
\hline 8 & Combine Fixations: Specific & $\begin{array}{l}\text { Combines two or more consecutive fixations } \\
\text { occurring on the same specific interface item } \\
\text { of interest }\end{array}$ \\
\hline
\end{tabular}

(1983), and it is a simplified version of algorithms used in bioinformatics (Thompson, Higgins, \& Gibson, 1994).

Example of use. After running the basic analyses, the user wishes to sequentially align general-level protocol paths that represent choice tasks. To perform the analysis, the example user applies the Revert function to desegment and reset the DMAP data to the point before filtering. First, the example ProtoMatch user removes fixations without assigned interface objects and cursor fixations. Second, she combines consecutive fixations into superdwells. Third, she resegments the protocols using the same segmentation keys used in the examples above: "((event jdm-start) (click choose))." Finally, the example user sequentially aligns each segment to all other segments within a log file by executing the Begin Sequencing function without entering a hypothesized segment. The result is a matrix of minimum-edit distances for each choice task relative to all other choice tasks for the same scenario that was saved to a file (see Table 4).

The minimum-edit matrix (Table 4) can be used to determine the prototypical protocol path of a user completing a single DMAP choice task. The prototypical protocol path is simply the choice task (segment) with the lowest average minimum-edit distance in comparison with all other choice tasks. Choice Task 11 in Table 4 is the prototypical protocol path for participant $\mathrm{S} 2$ for a single DMAP scenario. Notice that comparing Choice Task 1 to Choice Task 1 has a minimum-edit distance of 0 . Of course, including such 0 comparisons artificially deflates the average minimum-edit distances, but what is important is the ranking of the choice tasks based on their average minimum-edit distance, not the absolute value of the average minimum-edit distance.

To highlight the usefulness of prototypical protocol paths, prototypes were determined for participants S1 and S2 (see Table 5). First, notice that S2's protocol path contains 27 elements and S1's contains 20 elements. Next, notice that both participants begin their respective prototypical protocol paths in exactly the same way. It is on the sixth protocol (see Table 5) that participants begin to diverge in their prototypical protocol paths. That is, the participants execute the same basic behavioral elements (e.g., searching the radar and the ToA, clicking on radar targets, etc.), but in different orders. This example illustrates ProtoMatch's ability to highlight differences in task strategies, emphasizes that the same primitive operations of behavior are pieced together to form different general interactive routines (or strategies) in a given task environment, and pinpoints when and where the different routines diverge.

Although not all possible and worthwhile data analyses have been discussed above, the provided examples elucidate the power of ProtoMatch. Basic analyses provide simple measures that allow a surface-level perspective of behavior within a particular environment. However, ProtoMatch allows the user to go well beneath the surface. For instance, instead of dividing the log file into choice tasks, the example ProtoMatch user could have divided the log file into segments corresponding to eye fixations on the ToA and clicking on a target on the radar, effectively segmenting the log file into individual searches for choice alternatives in the radar. The example user could then execute the sequence alignment analyses on the newly segmented data to find the prototypical protocol path associated with finding ToA "Track \#s" in the radar. To get even deeper into the data, the user could keep all acquired fixations and only combine them into dwells rather than superdwells.

\section{GENERAL DISCUSSION}

ProtoMatch is a software tool designed to be flexible and general enough to be used in multiple task environments. It follows from the tradition of automating protocol analyses and takes such automation one step farther by sequentially integrating movement and fixed-location eye gaze and cursor protocols to produce protocol paths. Such protocol paths have already begun to shed light on behavior within dynamic, highly interactive, and visually complex task environments such as DMAP.

ProtoMatch provides several important extensions to exploratory analyses on eye gaze and cursor protocols: 


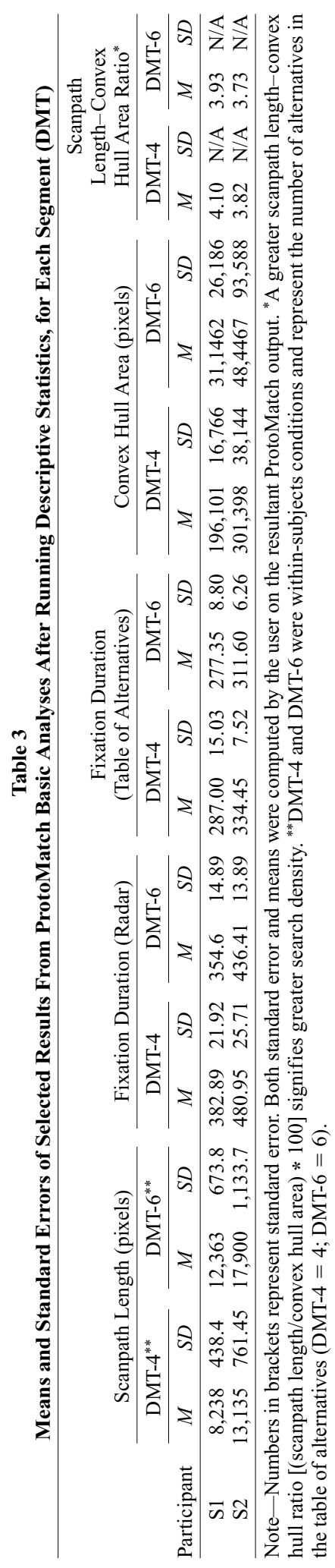

the abilities to easily combine, filter, and analyze sequential protocol data. Among the analyses available in ProtoMatch are two important functions that facilitate the analysis of large amounts of sequential protocol data: (1) a set of automated analyses adopted from the literature (Goldberg \& Kotval, 1999) and (2) a sequence analysis routine allowing the user to compute objectively the similarity between protocol paths, as was described above. The ability to compute the similarity between protocol paths objectively will certainly open doors to new behavioral analyses of high-density, high-definition data from within the same or different task environments and promises to facilitate understanding of strategy shifts in complex, dynamic, interactive tasks.

\section{Limitations}

A key limitation is the current inability to perform cluster-type analyses on the obtained protocol paths. For example, when determining the prototypical protocol path, there may actually be two protocol paths that fit the data equally well. That is, half of the obtained protocol paths may cluster around a particular prototypical protocol path, and the other half may cluster around a second one. This suggests that two competing strategies are occurring within the analyzed log file. The ability to analyze changes in the clusters over time (i.e., from a participant's first to final scenarios) will likely capture the evolution of strategies used by a participant. For instance, Choice Tasks 3, 13, 16, 17, and 18 in Table 4 all have high minimum-edit distance averages relative to the other choice tasks. Choice Tasks 16, 17, and 18 appear to be more different from the other choice tasks but very similar to each other. These three protocol paths may represent a shift in the participant's strategy that could have been captured using clustering analyses.

\section{Future Work}

Several other extensions to ProtoMatch form a large pool of possible extensions. First, we are considering including all of the measures proposed and developed by Goldberg and Kotval (1999) that assess fixation location and scanpaths. Including the probability vectors and transition matrices proposed by Ponsoda et al. (1995) would also be a worthwhile endeavor.

A second, and extremely important, extension to ProtoMatch would be the ability to analyze multiple log files during a single run. Currently, ProtoMatch is a one-fileat-a-time system. Adding the ability to execute the basic analyses for a set of several $\log$ files is currently being implemented, as is the ability to sequentially align sets of several $\log$ files. Another extension to the sequence alignment module would be to modify the module to include more efficient and powerful sequence alignment algorithms. Such modifications might include the ability to specify the costs associated with edit types (i.e., insertion, deletion, and replacement), so that the algorithm performs the minimum number of edits with the lowest overall cost. 
Table 4

A Minimum-Edit Matrix for a Single Scenario of Participant S2

\begin{tabular}{|c|c|c|c|c|c|c|c|c|c|c|c|c|c|c|c|c|c|c|c|}
\hline \multirow{2}{*}{$\begin{array}{c}\text { Choice } \\
\text { Task }\end{array}$} & \multicolumn{18}{|c|}{ Choice Task } & \multirow{2}{*}{$\begin{array}{c}\text { Mean } \\
\text { Minimum-Edit } \\
\text { Distance }\end{array}$} \\
\hline & 1 & 2 & 3 & 4 & 5 & 6 & 7 & 8 & 9 & 10 & 11 & 12 & 13 & 14 & 15 & 16 & 17 & 18 & \\
\hline 1 & 0 & 8 & 12 & 5 & 9 & 9 & 5 & 6 & 7 & 5 & 6 & 5 & 15 & 5 & 7 & 7 & 7 & 6 & 6.89 \\
\hline 2 & 8 & 0 & 6 & 6 & 7 & 5 & 6 & 6 & 6 & 7 & 4 & 7 & 9 & 5 & 7 & 13 & 13 & 12 & 7.06 \\
\hline 3 & 12 & 6 & 0 & 9 & 7 & 5 & 11 & 12 & 8 & 13 & 8 & 13 & 6 & 9 & 10 & 19 & 19 & 18 & 10.28 \\
\hline 4 & 5 & 6 & 9 & 0 & 8 & 6 & 2 & 5 & 6 & 6 & 5 & 5 & 12 & 4 & 6 & 10 & 10 & 9 & 6.33 \\
\hline 5 & 9 & 7 & 7 & 8 & 0 & 8 & 10 & 9 & 8 & 10 & 7 & 10 & 11 & 7 & 9 & 16 & 15 & 15 & 9.22 \\
\hline 6 & 9 & 5 & 5 & 6 & 8 & 0 & 8 & 9 & 6 & 8 & 3 & 8 & 6 & 6 & 9 & 14 & 14 & 13 & 7.61 \\
\hline 7 & 5 & 6 & 11 & 2 & 10 & 8 & 0 & 5 & 8 & 4 & 5 & 4 & 14 & 4 & 6 & 8 & 8 & 7 & 6.39 \\
\hline 8 & 6 & 6 & 12 & 5 & 9 & 9 & 5 & 0 & 8 & 5 & 6 & 3 & 15 & 5 & 7 & 7 & 7 & 7 & 6.78 \\
\hline 9 & 7 & 6 & 8 & 6 & 8 & 6 & 8 & 8 & 0 & 7 & 5 & 7 & 8 & 6 & 7 & 12 & 12 & 11 & 7.33 \\
\hline 10 & 5 & 7 & 13 & 6 & 10 & 8 & 4 & 5 & 7 & 0 & 5 & 2 & 14 & 4 & 7 & 6 & 6 & 5 & 6.33 \\
\hline 11 & 6 & 4 & 8 & 5 & 7 & 3 & 5 & 6 & 5 & 5 & 0 & 5 & 9 & 3 & 7 & 11 & 11 & 10 & 6.11 \\
\hline 12 & 5 & 7 & 13 & 5 & 10 & 8 & 4 & 3 & 7 & 2 & 5 & 0 & 14 & 4 & 7 & 6 & 6 & 5 & 6.17 \\
\hline 13 & 15 & 9 & 6 & 12 & 11 & 6 & 14 & 15 & 8 & 14 & 9 & 14 & 0 & 12 & 12 & 20 & 20 & 19 & 12.00 \\
\hline 14 & 5 & 5 & 9 & 4 & 7 & 6 & 4 & 5 & 6 & 4 & 3 & 4 & 12 & 0 & 8 & 10 & 10 & 9 & 6.17 \\
\hline 15 & 7 & 7 & 10 & 6 & 9 & 9 & 6 & 7 & 7 & 7 & 7 & 7 & 12 & 8 & 0 & 10 & 9 & 10 & 7.67 \\
\hline 16 & 7 & 13 & 19 & 10 & 16 & 14 & 8 & 7 & 12 & 6 & 11 & 6 & 20 & 10 & 10 & 0 & 1 & 1 & 9.50 \\
\hline 17 & 7 & 13 & 19 & 10 & 15 & 14 & 8 & 7 & 12 & 6 & 11 & 6 & 20 & 10 & 9 & 1 & 0 & 2 & 9.44 \\
\hline 18 & 6 & 12 & 18 & 9 & 15 & 13 & 7 & 7 & 11 & 5 & 10 & 5 & 19 & 9 & 10 & 1 & 2 & 0 & 8.83 \\
\hline
\end{tabular}

Note-Data were produced by using "Begin Sequencing" without entering a hypothesized sequence. Each cell represents the minimum number of edits (insertions, deletions, and replacements) necessary to change Choice Task 1 into Choice Task 2 ( 8 edits), into Choice Task 3 (12 edits), and so forth. Note that when a choice task is compared with itself, no edits are necessary. Boldface row represents the prototypical protocol path for the scenario.

A third possible extension would be to promote the current Fixatron static displays to dynamic reenactments. These reenactments would provide researchers with the ability to repeatedly view filtered segments in real time.

Table 5

A Comparison of the Two Best-Fitting Prototypical Protocol Paths for Participants S2 and S1

\begin{tabular}{|c|c|c|}
\hline \multirow[b]{2}{*}{ Protocol Number } & \multicolumn{2}{|c|}{ Prototypical Protocol Paths } \\
\hline & $\mathrm{S} 2$ & $\mathrm{~S} 1$ \\
\hline 1 & Eye data ToA & Eye data ToA \\
\hline 2 & Eye data radar & Eye data radar \\
\hline 3 & Click target & Click target \\
\hline 4 & Eye data ToA & Eye data ToA \\
\hline 5 & Eye data radar & Eye data radar \\
\hline 6 & Eye data ToA & Click target \\
\hline 7 & Eye data radar & Eye data ToA \\
\hline 8 & Eye data ToA & Eye data radar \\
\hline 9 & Eye data radar & Click target \\
\hline 10 & Click target & Eye data radar \\
\hline 11 & Eye data ToA & Eye data ToA \\
\hline 12 & Eye data radar & Eye data radar \\
\hline 13 & Click target & Click target \\
\hline 14 & Eye data ToA & Eye data ToA \\
\hline 15 & Eye data radar & Eye data radar \\
\hline 16 & Eye data ToA & Click target \\
\hline 17 & Eye data radar & Eye data ToA \\
\hline 18 & Click target & Click ToA \\
\hline 19 & Eye data ToA & Eye data choose \\
\hline 20 & Eye data radar & Click choose \\
\hline 21 & Eye data ToA & \\
\hline 22 & Eye data radar & \\
\hline 23 & Eye data ToA & \\
\hline 24 & Click ToA & \\
\hline 25 & Eye data ToA & \\
\hline 26 & Eye data choose & \\
\hline 27 & Click choose & \\
\hline
\end{tabular}

Note-ToA represents table of alternatives (right side, Figure 2). The boldface row represents the point of divergence in protocol paths between $\mathrm{S} 2$ and $\mathrm{S} 1$.
Real-time reenactments would serve as an exploratory tool for users to gain insights on how tasks are performed.

\section{CONCLUSIONS}

Reusable software tools for analyzing either cursor or eye movement protocol data are rare, and software tools that incorporate the two are rarer still, if they exist at all. Collecting protocol data is easy, but analyzing and interpreting the data are tedious, difficult, and time-consuming tasks. The present report presented a software tool that was developed to alleviate these problems.

ProtoMatch provides a relatively robust analytical tool with little overhead; that is, no complicated grammars need to be learned, no goal-subgoal task decompositions need to be refined, and no computational cognitive models need to be generated and tested. Although each of these functions is invaluable for understanding complex behavior, eliminating the necessity of them from protocol analysis is an achievement. ProtoMatch also increases objectivity when comparing scanpaths, cursorpaths, and protocol paths, as is demonstrated in the examples above.

ProtoMatch provides a very flexible analytical tool that currently facilitates exploration of data and supports confirmatory analyses via the sequencing and basic analyses modules. ProtoMatch can be used across multiple domains, such as HCI, visual search, decision making, and possibly even psycholinguistics, to name but a few.

\section{REFERENCES}

Anderson, J. R. (2002). Spanning seven orders of magnitude: A challenge for cognitive modeling. Cognitive Science, 26, 85-112.

Card, S. K., Moran, T. P., \& Newell, A. (1983). The psychology of human-computer interaction. Hillsdale, NJ: Erlbaum.

Ericsson, K. A., \& Simon, H. A. (1980). Verbal reports as data. Psychological Review, 87, 215-251. 
FU, W.-T. (2001). ACT-PRO action protocol analyzer: A tool for analyzing discrete action protocols. Behavior Research Methods, Instruments, \& Computers, 33, 149-158.

Goldberg, J. H., \& Kotval, X. P. (1999). Computer interface evaluation using eye movements: Methods and constructs. International Journal of Industrial Ergonomics, 24, 631-645.

GRAY, W. D. (2002). Simulated task environments: The role of highfidelity simulations, scaled worlds, synthetic environments, and microworlds in basic and applied cognitive research. Cognitive Science Quarterly, 2, 205-227.

Gray, W. D., Schoelles, M., \& Myers, C. W. (2004). Strategy constancy amidst implementation differences: Interaction-intensive versus memory-intensive adaptations to information access in decisionmaking. Paper presented at the 26th Annual Meeting of the Cognitive Science Society, Chicago.

KARSH, R., \& BREITENBACH, F. W. (EDS.) (1983). Looking at looking: Amorphous fixation measure. Hillsdale, NJ: Erlbaum.

LipPs, M., \& Pelz, J. B. (2004). Yarbus revisited: Task-dependent oculomotor behavior. Paper presented at the Fourth Annual Meeting of the Vision Sciences Society, Sarasota, FL.

Lohse, G., \& Johnson, E. (1996). A comparison of two process tracing methods for choice tasks. Organizational Behavior \& Human Decision Processes, 68, 28-43.

Moyle, M., \& Cockburn, A. (2003). The design and evaluation of a flick gesture for "back" and "forward" in Web browsers. Paper presented at the Fourth Australian User Interface Conference, Adelaide, Australia.

Newell, A., \& CARD, S. K. (1985). The prospects for psychological science in human-computer interactions. Human-Computer Interaction, 1, 2209-2242.

Payne, J. W., Bettman, J. R., \& Johnson, E. J. (1993). The adaptive decision maker. New York: Cambridge University Press.

Ponsoda, V., Scott, D., \& Findlay, J. M. (1995). A probability vector and transition matrix analysis of eye movements during visual search. Acta Psychologica, 88, 167-185.

Rayner, K., Liversedge, S. P., White, S. J., \& Vergilino-Perez, D.
(2003). Reading disappearing text: Cognitive control of eye movements. Psychological Science, 14, 385-388.

RitTer, F. E., \& LARKIN, J. H. (1994). Developing process models as summaries of HCI action sequences. Human-Computer Interaction, 9, 345-383.

Salvucci, D. [D.], \& Anderson, J. R. (2001). Automated eye-movement protocol analysis. Human-Computer Interaction, 16, 39-86.

Salvucci, D. D., \& Macuga, K. L. (2002). Predicting the effects of cellular-phone dialing on driver performance. Cognitive Systems Research, 3, 95-102.

SAnderson, P. M., \& Fisher, C. (1994). Exploratory sequential data analysis: Foundations. Human-Computer Interaction, 9, 251-317.

Shen, J., Reingold, E. M., \& Pomplun, M. (2000). Distractor ratio influences patterns of eye movements during visual search. Perception, 29, 241-250.

Siegler, R. S., \& Stern, E. (1998). Conscious and unconscious strategy discoveries: A microgenetic analysis. Journal of Experimental Psychology: General, 127, 377-397.

Simon, H. A. (1999). The sciences of the artificial. Cambridge, MA: MIT Press.

Thompson, J. D., Higgins, D. G., \& Gibson, T. J. (1994). CLUSTAL $\mathrm{W}$ : Improving the sensitivity of progressive multiple sequence alignment through sequence weighting, position-specific gap penalties and weight matrix choice. Nucleic Acids Research, 11, 4673-4680.

ToDD, P., \& BenBASAT, I. (1994). The influence of decision aids on choice strategies: An experimental analysis of the role of cognitive effort. Organizational Behavior \& Human Decision Processes, 60, 36-74.

TodD, P., \& Benbasat, I. (1999). Evaluating the impact of DSS, cognitive effort, and incentives on strategy selection. Information Systems Research, 10, 357.

ToDD, P., \& BENBASAT, I. (2000). Inducing compensatory information processing through decision aids that facilitate effort reduction: An experimental assessment. Journal of Behavioral Decision Making, 13, 91-106.

YARBUs, A. F. (1967). Eye movements and vision. New York: Plenum. 


\section{APPENDIX \\ Log and Secondary-Object File Descriptions and Formats for ProtoMatch}

This appendix provides examples of $\log$ and secondary-object files necessary to run ProtoMatch. We begin with log file format and finish with secondary-object files.

\section{Log Files}

Before using ProtoMatch, and possibly before even collecting data, the format and information available in log files must be considered. ProtoMatch is currently completely dependent on the log file format, and a potential user must adhere to the $\log$ file format described below and in Table A1. The log files must be tab delimited and use predefined process delimiters in order for ProtoMatch to properly sort and use relevant behavioral and task environment information. Such process delimiters include system, interface object, and gaze and cursor delimiters. Table A1 presents an example of the necessary log file format and information and will be discussed in detail below.

The two process delimiters of highest importance are the system and eyegaze delimiters. System delimiters (see "System" rows, Table A1) include two or more fields that contain task time, system action, and other task-relevant records. Eyegaze delimiters include six fields containing task time, data quality (typically $1=$ good and $0=b a d$ ), pupil diameter, the $x$-coordinate of gaze position, the $y$-coordinate of gaze position, and cursor position information.

The inclusion of task-relevant interface objects in the log file allows ProtoMatch to be used in dynamic task environments. Including changes in interface objects in the $\log$ file enables ProtoMatch to update interface object information. For example, when ProtoMatch reads a line containing the text "TARGET $<$ tab $>$ $26186410<\mathrm{tab}>$ PERIODIC $<$ tab $>28<\mathrm{tab}>475<\mathrm{tab}>100<\mathrm{tab}>$ Radar" (see first "Target" row, Table A1), Target 28 is searched for in a list of stored interface objects. If Target 28 is found, it is updated accordingly; however, if it is not found, a new target is created, labeled " 28 ," and assigned initial Cartesian coordinates $(475,100)$ and a general area of "Radar."

Table A1

An Example of the Necessary Information and Formatting for Log Files Used by ProtoMatch

\begin{tabular}{lclcccc}
\hline $\begin{array}{c}\text { Process } \\
\text { Delimiter }\end{array}$ & Time & Descriptor & $\begin{array}{c}\text { Eye: Pupil Diameter } \\
\text { System \& } \\
\text { Target: Descriptor }\end{array}$ & $\begin{array}{c}\text { Eye or } \\
\text { Cursor: } x\end{array}$ & $\begin{array}{c}\text { Eye or } \\
\text { Cursor: } y\end{array}$ & $\begin{array}{c}\text { Cursor: }(x, y) \\
\text { System } \boldsymbol{\&} \\
\text { Target: General Area }\end{array}$ \\
\hline System & $\mathbf{2 6 1 8 6 3 9 4}$ & Add-Object & $\mathbf{1 4}$ & $\mathbf{6 0}$ & $\mathbf{2 0}$ & Radar \\
Target & $\mathbf{2 6 1 8 6 4 1 0}$ & Periodic & $\mathbf{2 8}$ & $\mathbf{4 7 5}$ & $\mathbf{1 0 0}$ & Radar \\
Eyegaze & 26186426 & 1 & 143 & 305 & 324 & $(300405)$ \\
Eyegaze & 26186442 & 1 & 145 & 308 & 320 & $(300405)$ \\
Eyegaze & 26186458 & 0 & Nil & Nil & Nil & $(300405)$ \\
Target & $\mathbf{2 6 1 8 6 4 7 4}$ & Periodic & $\mathbf{1 6}$ & $\mathbf{3 0 9}$ & $\mathbf{3 2 2}$ & Radar \\
Eyegaze & 26186490 & 1 & 140 & 309 & 321 & $(300405)$ \\
System & $\mathbf{2 6 1 8 6 5 0 6}$ & Add-Object & $\mathbf{3 8}$ & $\mathbf{6 5 4}$ & $\mathbf{1 5 3}$ & Radar \\
Cursor & 26186522 & Nil & Nil & 303 & 400 & \\
\hline
\end{tabular}

Note-ProtoMatch data must be formatted in tab-delimited columns. Data for system and target delimiters are presented above in bold. The first column contains the process delimiter, the second always contains the time stamp, and the third is some type of descriptor. The fourth column contains one of the following: pupil diameter for eyegaze delimiters or a numeric descriptor for system and target delimiters. The fifth and sixth columns contain $(x, y)$ coordinates for all except system delimiters, in which case the columns contain more descriptors. The last column contains cursor positions for eyegaze delimiters [formatted with parentheses and a space but without a comma: e.g., (303 405)] and general areas of interest for system or target delimiters (e.g., Radar). 


\section{APPENDIX (Continued)}

\section{Secondary-Object Files}

Secondary interface objects are areas of the interface whose relevant information (exact display location, object label, and general display location) has not been included in the $\log$ file. Secondary-object files are not necessary to use ProtoMatch, but if interface areas of interest, specific or general, are not included in the log file, this feature provides a way to include these areas. Likewise, if ProtoMatch is used with a static task environment, interface objects do not need to be included in the $\log$ file, and only a secondary-object file is needed to provide all interface objects of interest.

Just like the log files, secondary-object files must be tab-delimited text files and strictly adhere to a predefined format. Table A2 demonstrates the necessary format for creating a secondary-object file.

Table A2

An Example of Necessary Information and Formatting for Secondary Interface Object Files Used by ProtoMatch

\begin{tabular}{cccccc}
\hline $\begin{array}{c}\text { Object } \\
\text { Identifier }\end{array}$ & $\begin{array}{c}\text { Specific } \\
\text { Object Label }\end{array}$ & $\begin{array}{c}\text { Object: } x \text {-coord. } \\
\text { General: } \\
\text { Smallest } \boldsymbol{x} \text {-coord. }\end{array}$ & $\begin{array}{c}\text { Object: } \boldsymbol{y} \text {-coord. } \\
\text { General: } \\
\text { Largest } \boldsymbol{x} \text {-coord. }\end{array}$ & $\begin{array}{c}\text { Object: Label/Location } \\
\text { General: } \\
\text { Smallest } \boldsymbol{y} \text {-coord. }\end{array}$ & $\begin{array}{c}\text { General: } \\
\text { Largest } \boldsymbol{y} \text {-coord. }\end{array}$ \\
\hline 1 & Alt1 & 784.5 & 308.5 & DMT & \\
2 & Alt2 & 784.5 & 357.5 & DMT \\
3 & Alt3 & 784.5 & 406.5 & DMT \\
4 & Alt4 & 784.5 & 455.5 & DMT \\
5 & Alt5 & 784.5 & 504.5 & DMT \\
6 & Alt6 & 784.5 & 553.5 & DMT \\
7 & Time & 4 & 468 & Time & \\
8 & Score & 4 & 758 & Score & \\
9 & Ownship & 256 & 760 & Ownship & \\
10 & Choose & 792 & 692 & Choose & \\
General & Radar & $\mathbf{0}$ & $\mathbf{5 1 1}$ & $\mathbf{2 2}$ & $\mathbf{7 6 8}$ \\
General & DMT & $\mathbf{7 5 9}$ & $\mathbf{8 1 0}$ & $\mathbf{2 8 4}$ & $\mathbf{6 7 5}$ \\
\hline
\end{tabular}

Note-ProtoMatch data must be formatted in tab-delimited columns. Data for general areas of interest are presented above in bold. The first column contains an identifier for the secondary interface object or "General" for a general area of interest. The second column contains a label for the object or area of interest. Columns 3 and 4 contain the $(x, y)$ coordinates for an object or the smallest and largest $x$-coordinates for a general area. Column 5 for an object contains the location of an object or repeats the label from Column 2; for an area of interest, Columns 5 and 6 contain the smallest and largest $y$-coordinates for the area.

(Manuscript received November 15, 2004; revision accepted for publication January 31,2005 .) 